\title{
Tangence
}

\section{Sciences, histoire des sciences et littérature}

\section{Gilles Denis}

Numéro 61, décembre 1999

Savoir et littérature

URI : https://id.erudit.org/iderudit/008163ar

DOI : https://doi.org/10.7202/008163ar

Aller au sommaire du numéro

Éditeur(s)

Presses de l'Université du Québec

ISSN

0226-9554 (imprimé)

1710-0305 (numérique)

Découvrir la revue

Citer cet article

Denis, G. (1999). Sciences, histoire des sciences et littérature. Tangence, (61),

8-33. https://doi.org/10.7202/008163ar d'utilisation que vous pouvez consulter en ligne.

https://apropos.erudit.org/fr/usagers/politique-dutilisation/ 


\section{Sciences, histoire des sciences et littérature Gilles Denis, Équipe REHSEIS (CNRS et Université Denis Diderot)}

On peut constater différents niveaux d'analyse selon les études portant sur les rapports entre littérature et science(s). Il s'agit dans certains cas de mettre l'accent sur la présence de la science dans une œuvre (Gustave Flaubert, Jules Verne, Aldous Huxley, etc. ${ }^{1}$ ). À d'autres occasions, c'est l'utilisation de la science par la littérature (analogie, métaphore, méthode de construction de l'intrigue, psychologie des personnages, etc. ${ }^{2}$ ) qui est le thème de l'analyse. Parfois, on s'intéresse à la comparaison des deux approches, scientifique et littéraire, et on s'interroge sur l'existence de deux sensibilités, "deux cultures" ${ }^{3}$. Dans un dernier cas, la littérature est élargie explicitement ou implicitement aux humanités, et on aborde la concurrence entre celles-ci et les sciences dans la capacité à former l'individu, à l'aider à appréhender le monde et à vivre avec lui (éthique, connaissance, morale, politique, etc. ${ }^{4}$ ).

1. Voir notamment dans Danielle Jacquart (dir.), De la science en littérature à la science-fiction, Paris, Éditions du CTHS, 1996: Chantal Connochie-Bourgne, "Lancelot et le tempérament colérique", p. 11-22; Daniel Compère, "Le jeu avec les références scientifiques dans les romans de Jules Verne", p. 137145; Suzanne Débarbat et Simone Dumont, "Sur quelques exemples de la présence de l'astronomie dans la littérature, le dessin humoristique et la bande dessinée", p. 167-183.

2. Voir notamment Katherine Hayles, Chaos Bound. Orderly Disorder in Contemporary Literature and Science, Ithaca, Cornell University Press, 1990; Daniel Albright, Quantum Poetics. Yeats, Pound, Eliot and the Science of Modernism, New York, Cambridge University Press, 1997; Marie Madeleine Fontaine, "Quaresmeprenant. L'image littéraire et la contestation de l'analogie médicale", 1984, repris dans Libertés et savoirs du corps à la Renaissance, Caen-Orléans, Paradigme, 1993, p. 195-220.

3. Voir notamment Aldous Huxley, Literature and Science, New York, Harper and Row, 1963; Joseph W. Slade et Judith Yaross Lee, Beyond the Two Cultures. Essays on Science, Technology, and Literature, Ames, Iowa State University Press, 1990; Stuart Peterfreund, Literature and Science, Boston, Northeastern University Press, 1990; John Brockman, The Third Culture, New York et Toronto, Simon and Schuster, 1995; Paul Braffort, Science et littérature, les deux cultures, dialogues et controverses pour l'an 2000, Paris, Diderot Multimedia, 1998.

4. Voir notamment le débat Thomas Huxley/John Burroughs et Mattew Arnold. Les textes de Huxley et Burroughs se trouvent dans Martin Gardner, The 
10

L'histoire des sciences participe aux débats sur les caractéristiques de la science, de l'activité scientifique, ses a priori, ses conséquences. Privilégiant la science qui se fait réellement dans l'histoire (avec les précautions d'usage sur la "méthode" historique) différente de celle imaginée par les savants et les philosophes, elle induit parfois certaines prises de position idéologiques sur la nature de la science. Nous présentons ici quelques réflexions sur les rapports entre littérature et science(s) à la lumière des débats existant en histoire des sciences. Nous montrons aussi, par quelques exemples précis, comment les résultats de l'histoire des sciences peuvent servir à l'analyse littéraire.

\section{Vous avez dit « deux cultures »? Mais comment?}

Dans un de ses Problemi, Galilée raconte que les brouillards et les rosées sont constitués de minuscules "étoiles" d'eau. Il invite à les voir sur les toiles d'araignée, autour des haies, après un brouillard, ou le matin de bonne heure, après la rosée de la nuit, avant que le Soleil ne les consume ${ }^{5}$. Galilée tente ensuite d'expliquer une maladie des plantes appelée "ustilago" en latin et "brûlure" en français :

Or il advient que quelquefois, après un brouillard et le Soleil se découvrant, les feuilles de vigne et d'autres feuillages deviennent arides et se dessèchent tout à fait. La cause d'un tel effet est la suivante. Se pose, (pendant le temps que dure le brouillard), sur les feuilles de vigne, une très grande quantité de ces étoiles très menues qui rendent perceptibles les toiles d'araignée; ces [étoiles] sont de forme ronde et parfaitement sphérique; se dissout ensuite le brouillard et se découvre le Soleil, les rayons duquel, passant à travers ces très petites sphères, frappent, par réfraction, la feuille, qui leur est sous-jacente; et de la même manière que ces mêmes rayons, passant au travers d'une boule de cristal ou d'une carafe pleine d'eau, et, frappant sur de l'amadou et sur du drap, ou autre chose similaire, les réchauffent et les brûlent; ainsi, de la même façon, passant par

Sacred Beetle and Other Great Essays in Science, Buffalo, N. Y., Prometheus Books, 1984, p. 131 et 149 et celui de Arnold dans David Cornelius et Edwin St. Vincent, Cultures in Conflict. Perspectives on the Snow-Leavis Controversy, Scott, Foresman and $\mathrm{C}^{\circ}, 1964$, p. 78.

5. Galileo Galilei, Opere, tomo terzo, nuovo edizione, Firenze, Gaetano Tartini e Santi Franchi, 1718; cf. "Problemi varj di Galileo Galilei", Problema VI, p. 52 . 
ces petits globules, ils viennent à réchauffer tellement la feuille qu'ils lui retirent l'eau et la dessèchent absolument. ${ }^{6}$

La théorie du transformisme mise en place par Lamarck prend appui dans ses Recherches sur l'organisation des corps vivants, texte édité en 1802, sur des exemples de ce genre:

De même l'on sent que l'oiseau de rivage, qui ne se plait point à nager, et qui cependant a besoin de s'approcher des bords de l'eau pour y trouver sa proie, sera continuellement exposé à s'enfoncer dans la vase. Or cet oiseau voulant faire en sorte que son corps ne plonge pas dans le liquide, fait tous ses efforts pour étendre et allonger ses pieds. ${ }^{7}$

L'origine des espèces de Darwin ${ }^{8}$ présente une approche et un style peu différents.

De nos jours, la lumière excite les molécules:

La capacité d'absorption de la lumière par une molécule dépend de l'arrangement des électrons autour des noyaux constituant sa structure. Lorsqu'un photon est absorbé, un électron est amené à un niveau d'énergie plus élevé. Ce phénomène se produit selon une règle du tout ou rien; pour être absorbé, le photon doit renfermer une quantité d'énergie (un quantum) qui corresponde exactement à celle de la transition électronique. Une molécule ayant absorbé un photon est dans un état excité, généralement instable. ${ }^{9}$

Le texte peut sembler plus "scientifique"! Pourtant c'est un énoncé constitué de propositions ayant les mêmes caractéristiques logiques et grammaticales que les précédents. Le photon a remplacé l'oiseau de rivage et la molécule, la vase. Apprenons à voir ce petit photon exciter la belle molécule et le texte nous semblera tout aussi "littéraire" !

À l'occasion d'un colloque récent de néphrologie pédiatrique, on a pu prendre connaissance du texte suivant, somme toute banal en un tel lieu, d'une pédiatre de l'hôpital des enfants malades de Paris :

6. Galileo Galilei, ibid., Problema VII, p. 52. Notre traduction.

7. Lamarck, Recherches sur l'organisation des corps vivants, Paris, Fayard, 1986 (Paris, Maillard, 1802/An X), p. 48.

8. Charles Darwin, L'origine des espèces, Paris, Flammarion, 1992.

9. Albert L. Lehninger et David L. Nelson, Principes de biochimie, Paris, Flammarion, 1994, p. 574 . 
La survenue d'une glomérulopathie de type paranéoplasique précédant ou accompagnant le diagnostic d'une hémopathie est classique mais peu fréquente. Nous rapportons l'association entre une GNMP [Glomérulonéphrite membrano-proliférative] et une myélodysplasie survenue chez une enfant de cinq ans. Claire, sans antécédent, présente, sept jours après une angine, une hématurie macroscopique, une insuffisance rénale aiguë (oligo-anurie, créat $=256 \mu \mathrm{mol} / \mathrm{l}, \mathrm{K}=4,6 \mathrm{mmol} / \mathrm{l}, \mathrm{Hb}=79 \mathrm{~g} / \mathrm{l}$, plaquettes $=39000, \mathrm{~GB}=5200$, shizo $=0$ ) faisant suspecter un SHU atypique. Le bilan met en évidence une thrombopénie périphérique (Ac anti-plaquettes, présence de mégacaryocites au myélogramme de J3), une diminution du C3 (0,58 g/l), la présence d'un C3Nef, des auto-Ac (anti-muscle lisse, AAN, anticardiolipides), une hypergammaglobulinémie (lgG 19,4g/l, IgA $9,3 \mathrm{~g} / \mathrm{l}$, IgM 2,35 g/l), une infection à CMV (sérologie positive en IgM). L'évolution rénale est favorable sous dialyse péritonéale et traitement symptomatique mais il persiste une hématurie, une protéinurie et une thrombopénie. Une PBR est réalisée un mois et demi après avoir débuté une corticothérapie qui corrige transitoirement la thrombopénie. Elle révèle une GNMP atypique. ${ }^{11}$

Ces quatre textes appartiennent à la littérature scientifique. Les deux premiers semblent de prime abord pouvoir être plus aisément qualifiés de "récit littéraire" mais les deux autres peuvent être rapprochés de certains textes littéraires hermétiques postmodernes. Le premier constat quasiment banal posé par un historien des sciences est celui de la très grande diversité des textes savants, illustrée par nos quatre exemples, variant selon les disciplines, les époques, les objets étudiés, les traditions intellectuelles et institutionnelles. Doit-on suivre certains auteurs qui séparent plus ou moins nettement le discours littéraire du discours savant (ou qui discutent de cette séparation) mais en plaçant, ce qu'ils ne font généralement pas, la frontière au sein même de la science, ou bien encore suivre d'autres auteurs qui proposent d'opposer d'un côté la technologie (dont la médecine?) et, de l'autre, la science et les arts ${ }^{11}$ ? En guise de résultat du choix de telles oppositions, on peut alors se représenter, comme Medawar, deux excès: une tendance "scientiste" renvoyant la littérature d'imagination du côté de

10. Christine Piètrement et al., Glomerulonéphrite membrano-proliférative révélatrice d'une myélodysplasie chez une enfant de 5 ans, Résumé, Congrès annuel de la Société de néphrologie pédiatrique, Luxembourg, 1998.

11. Voir Martha Turner, Mechanism and the Novel. Science in the Narrative Process, New York, Cambridge University Press, 1993, p. 8-21; Wylie Sypher, Literature and Technology. The Alien Vision, New York, Random House, 1968. 
l'industrie du spectacle et une tendance "poétiste" déniant à la littérature savante une capacité à comprendre le sens profond du Livre de la Nature ${ }^{12}$. En fait de telles appréciations prennent en partie leur source dans la façon dont certains "savants" et certains "poètes" conçoivent leur activité et leur production mais pas, selon nous, dans l'analyse de celles-ci. Peut-on plutôt décrire le champ de la "culture" comme "un continuum avec le purement conceptuel à une extrémité, le purement perceptuel à l'autre " ${ }^{13}$ en positionnant nos textes savants en différents lieux plus ou moins éloignés sur ce continuum? Mais à quoi ressemblent le "purement conceptuel" et le "purement perceptuel" et comment évaluer la part relative du "conceptuel" et du "perceptuel" dans le cas où ils coexisteraient? Le Cercle de Vienne et les empiristes logiques du $\mathrm{xx}^{\mathrm{e}}$ siècle, après avoir désespérément tenté de le faire, ont abandonné la quête d'une définition de l'énoncé scientifique et d'une démarcation entre celui-ci et l'énoncé non scientifique ${ }^{14}$. Ainsi, la notion de science (et de littérature?) apparaît floue et l'appréhension de la nature de celle-ci, de l'activité scientifique, ou de ses capacités et de ses résultats, semble sans cesse s'éloigner parce que cette notion est d'abord le résultat d'une histoire humaine avant de s'appuyer sur une définition logique. L'analyse de la comparaison entre science et littérature nous semble donc d'entrée de jeu inadaptée, inefficace et dépourvue de sens. La controverse modèle Snow/Leavis sur les "deux cultures" porte en fait essentiellement sur la part relative dans l'enseignement des sciences et des humanités et renvoie implicitement aux inquiétudes des enseignants, étudiants et parents ainsi qu'aux choix politiques en la matière.

Nous ne pensons pas que l'on puisse comparer science(s) et littérature en insistant sur leur forme d'expression ${ }^{15}$, en disant par exemple que les deux font appel à des métaphores, comme il ne

12. Peter B. Medawar, The Hope of Progress. A Scientist Look at Problems in Philosophy, Literature and Science, Garden City, N. Y., Anchor Books, 1973; cf. "Science and Literature".

13. George Guffey et George Slusser, "Literature and Science", dans Jean-Pierre Barricelli et Joseph Gibaldi, Interrelations of Literature, New York, Modern Language Association, 1982.

14. Voir Pierre Jacob, De Vienne à Cambridge. L'héritage du positivisme logique, Paris, Gallimard [1980], 1996.

15. Voir Frederick Amrine, Literature and Science as Modes of Expression, Dordrecht, Boston et Londres, Kluwer Academic Publishers, 1989. 
14

nous semble pas pertinent d'affirmer vouloir, de la même manière, comparer religion(s) et littérature. Il n'est pas plus étonnant que la science fasse appel à des métaphores qu'elle se serve de prédicats, de phrases, etc., puisque le discours scientifique est d'abord littéraire en ce qu'il est travail d'écriture, qu'il décrit, exprime des idées, des sensations, qu'il est le résultat de choix sur le fond et la forme dans le but de se faire comprendre, de plaire et de convaincre. La science au sens large (recherche, formation, enseignement, vulgarisation, administration, chercheur, équipe, etc.) tourne autour du texte (présentation des hypothèses, des projets, des résultats, etc.). Il peut y avoir de la science dans la littérature (des concepts, des théories, un vocabulaire issus de la science, etc.) comme il peut y avoir de l'agriculture, de la chasse, de l'érotisme ou de la dentelle mais le texte littéraire en revanche n'appartient ni à la science ni au discours scientifique. Le discours (l'écriture) scientifique peut et doit être étudié, analysé comme tout autre écrit littéraire ${ }^{16}$.

\section{Mais où est la science?}

Peut-on rapprocher ce qui a été appelé la "science en action" et la "science achevée" respectivement de l'expérience vécue, ressentie et pensée de l'écrivain, et du récit et de la fiction qui en découlent. La "science en action", comme l'expérience vécue, ressentie et pensée de l'écrivain sont difficilement accessibles à l'approche historique et à l'analyse. L'analyse littéraire s'intéresse à la génétique des ouvres et au processus créateur, mais l'essentiel de la littérature reste le texte achevé. Le terme de science sousentend en revanche tout à la fois les textes finis et les processus qui ont abouti à ceux-ci intégrant la formation du chercheur, ses moyens financiers, techniques, institutionnels, etc. Pour l'historien ou le philosophe des sciences, se pose la question de ce qui est essentiel dans la science et la création scientifique, la "science en action" ou la "science achevée". Les carnets de laboratoire appartiennent à la première et leur étude montre le caractère confus,

16. Peter Dear, The Literary Structure of Scientific Argument. Historical Studies, Philadelphie, University of Pennsylvania Press, 1991; Marcello Petra, Persuading Science. The Art of Scientific Rhetoric, Canton, Maine, Science History Publication, 1991; David Locke, Science as Writing, New Haven et Londres, Yale University Press, 1992; Vincent de Coorebyter, Rhétoriques de la science, Paris, Presses universitaires de France, 1994. 
alogique, imprévisible de la démarche scientifique. Les articles scientifiques appartiennent à la seconde. Ils expriment des résultats présentés au contraire comme ceux d'une méthode claire, logique et prévisible, reconstruite a posteriori. Répondre dans un sens ou dans un autre peut révéler une position idéologique, disciplinaire et/ou institutionnelle. Prétendre que la science, c'est avant tout la "science en action", la science qui se fait réellement au jour le jour, signifie mettre l'accent sur son historicité, sa contingence et son contexte, la "science achevée" pouvant n'être alors appréciée que comme une reconstruction imaginaire, mythique et poétique sans signification autre que sociale ("intersubjectivité" collective) de la méthode suivie pour l'obtention des résultats. Dans ce cadre, on peut à l'extrême considérer que la nature, résultat du processus scientifique, pourrait être autre. Le texte achevé, la théorie explicative proposée peuvent alors être assimilés par certains historiens, sociologues ou philosophes des sciences à la partie d'un mythe ou d'une religion, celui ou celle de l'Occident moderne et contemporain (une "physicogonie" expliquant le monde et la nature comme tant d'autres). S'intéresser essentiellement à la "science achevée", c'est minimiser le processus précédant la mise en forme logique des résultats, la "découverte" ne s'exprimant véritablement que dans celle-ci. L'essentiel de la science, de l'activité scientifique n'est pas l'expérience particulière, vécue, ressentie et pensée du savant ou de son équipe, perceptible notamment dans les carnets de laboratoire, mais le passage à "l'universel" par la mise en forme - la reconstruction - logique dans les articles scientifiques ou les manuels scolaires. Que signifie alors une fois encore la mise en rapport de la science et la littérature? Parle-t-on de la "science en action", de la "science achevée", de l'activité scientifique (mais laquelle, celle au jour le jour ou celle qui met en forme), de la littérature scientifique dans son ensemble, carnets de laboratoire, brouillons compris, ou réduite aux articles et ouvrages diffusés? Parle-t-on de la science au sens large quand on regroupe sous ce terme tout à la fois les écrits, les hommes, les processus et méthodes, les moyens techniques et financiers, les institutions de recherche, de décision et d'enseignement, les choix politiques et socio-économiques? L'histoire des sciences, ces dernières années, généralement, quelles que soient d'ailleurs les écoles de pensée, réduit de moins en moins la science à ses écrits achevés, mais considère la science au sens large, aucun élément, dont ces textes achevés, ne pouvant être dissocié des autres. 
16

Sokal et Bricmont accusent avec ironie l'usage abusif et non pertinent dans la littérature, notamment post-moderne au sens large, de métaphores construites à partir du langage de la science. Ils reprochent en effet aux auteurs de celles-ci de ne pas véritablement comprendre et maîtriser les théories scientifiques qu'ils évoquent. Il n'y a pas de doute, cependant, qu'il y a plus grave, et c'est lorsque la littérature reprend avec une bonne compréhension et une bonne pertinence le discours et les résultats de la science, mais Sokal et Bricmont, croyants de celle-ci et de sa marche progressive vers la "connaissance" de la réalité, ne peuvent s'y arrêter. En effet, la littérature, malgré les critiques, s'est souvent faite l'alliée de la science conquérante. Elle a repris, habituellement rapidement après leur production, les modèles explicatifs, les théories, les diffusant auprès du grand public, favorisant tel camp dans une controverse, telle école, telle tradition. Que l'on songe à Aldous Huxley et au darwinisme. Le texte littéraire aide la science à faire passer ses modèles, ses concepts, son vocabulaire, sa "réalité", sa "nature" dans le langage commun. Le parasite, le microbe, le gène, l'énergie, le magnétisme, pour ne nommer que ces derniers, prennent ainsi de plus en plus de consistance. Parfois, la littérature ou l'analyse littéraire accuse un temps de retard, reprenant et s'appuyant sur des concepts d'une époque révolue et abandonnés à la nôtre. C'est le cas de la notion de vie, principe mystérieux, objet de recherche et de spéculation scientifiques particulièrement au début du $\mathrm{xIX}^{\mathrm{e}}$ siècle, à l'origine de la construction du terme "biologie". Les tendances physicalistes réductionnistes de la seconde moitié du $\mathrm{XIX}^{\mathrm{e}}$ siècle ont abouti au $\mathrm{xx}^{\mathrm{e}}$ siècle à mettre hors de la biologie le concept de vie, dans le sens où l'on ne retient plus l'idée qu'il existerait des principes vitaux étrangers à la physique et à la chimie. Pourtant, Thomas Narcejac, dans une analyse du roman policier publiée en 1975, une trentaine d'années après le début de la biologie moléculaire, reprend ce concept et ce débat qui n'ont plus cours:

Les synthèses que la vie obtient sans effort, grâce à un principe mystérieux, jamais la science, armée de toutes les ressources de la physicochimie, ne parviendra à les réaliser alors qu'elle connaît pourtant leurs éléments. Et ce qui est vrai, au niveau de la biologie, l'est aussi au niveau de la littérature. ${ }^{17}$

17. Thomas Narcejac, Une machine à lire. Le roman policier, Paris, Denöel et Gonthier, 1975, p. 18. 
La littérature a plus fondamentalement propagé l'idée de la capacité de la science à représenter et à maîtriser la réalité qui nous entoure, perceptible ou cachée.

La science qui règle et fixe les saisons,

Qui du vaste univers enseignant la structure,

Et des astres errants la marche toujours sûre. ${ }^{18}$

Sans être aussi caricaturale que La sphère de Dominique Ricard (1741-1803), la littérature s'est souvent placée, implicitement, du côté du choix de la "science achevée", science "universalisée" comme activité centrale, essentielle de l'activité scientifique, qui exprimerait ainsi le "réel", le "vrai", prenant position idéologiquement et philosophiquement sur la nature de la science sans en être généralement consciente. Nous ne récusons pas ici "l'universalité" de la science (nous avons tendance à la retenir) ou ses capacités à dévoiler le "réel" ou une part du "réel". Nous faisons remarquer que les auteurs littéraires très généralement, quelle que soit la forme qu'ils adoptent pour s'exprimer, s'ils critiquent ou même rejettent la science, son approche ou ses conséquences, optent implicitement pour une telle représentation de la nature de la science. Nous observons que la littérature, mais aussi dans une moindre mesure ses commentateurs qui étudient ses rapports avec la science (tous ne sont pas Medawar ${ }^{19}$ ) ne prend nécessairement pas en compte le débat posé ici, commun en histoire et philosophie des sciences, en adhérant à une approche "réaliste" et "universaliste" naïve, même (d'autant plus?) colorée par les tendances du vingtième siècle s'intéressant à l'imprévisibilité, au chaos, à la complexité, à l'autorégulation ou la rétroaction.

\section{La littérature de science-fiction}

Le roman de science-fiction, particulièrement analysé dans le cadre des études sur les rapports entre science et littérature,

18. Dominique Ricard, La sphère. Poème en huit chants, Paris, Le Clerc, 1796, an $\mathrm{V}$.

19. Peter B. Medawar, op. cit. Voir aussi Martha Turner, op. cit., p. 21. Georges Lewis Levine, Realism and Representation. Essays on the Problems of Realism in Relation to Science, Literature and Culture, Madison, Wisconsin, University of Wisconsin Press, 1993. John Christie et Sally Shuttleworth, Nature Transfigured. Science and Literature 1700-1900, Manchester (UK) et New York, Manchester University Press, 1989. 
s'avère un bon exemple de l'influence de cette dernière en faveur d'une certaine représentation de la science, de son histoire, de l'activité scientifique et de ses résultats. Le futur apparaît alors comme l'aboutissement d'une concaténation linéaire issue de notre présent. Les autres mondes, quels que soient les succès des efforts de l'imagination, ne sont guère que des quasi répliques du nôtre. On y manipule mieux et plus les gènes mais ce sont des gènes; on joue avec l'espace-temps mais c'est l'espace-temps; on y rencontre des microbes et des parasites extraordinaires, des atomes, des particules, des forces et des énergies magnétiques, des gravitations différentes de celle de la terre mais ce sont des microbes et des parasites, des atomes, des particules, des forces, des énergies, du magnétisme et des gravitations. L'histoire des sciences est ainsi implicitement perçue dans cette littérature, comme une marche ampliative, plus ou moins linéaire et régulière, vers la connaissance du "réel". La science dévoilerait peu à peu, par petits pans, la réalité. Ce qui est dévoilé est un acquis, une part de la connaissance. Cette façon implicite de concevoir l'histoire des sciences correspond à une représentation remise en cause bien qu'encore largement présente chez les scientifiques et le grand public. Les historiens des sciences contemporains ont très généralement une autre vision.

Prenons l'exemple de la biologie moléculaire des années soixante-dix. Elle n'est pas issue simplement et directement de la génétique du début du $\mathrm{xx}^{\mathrm{e}}$ siècle. Dans les années trente et quarante, les biochimistes cherchaient dans une protéine l'explication de la transmission héréditaire des caractères. Celle-ci, telle une enzyme, devait orienter le métabolisme vers telle ou telle expression phénotypique. On parle de cette période comme celle de la théorie enzymatique de la vie. Avery est considéré aujourd'hui par les biologistes comme celui ayant mis en évidence, en 1944, le rôle de l'ADN (acide désoxyribonucléique) dans le processus héréditaire. Il cherchait pourtant à démontrer celui de la protéine associée, prêtant à l'ADN une fonction énergétique similaire à celle des ATP (molécules d'adénosine triphosphate qui servent de source d'énergie pour les réactions du métabolisme). Il a longtemps soutenu que ses résultats, contraires à ses attentes, étaient perturbés par des problèmes de contamination par la protéine. Avec le développement des théories de l'information, l'arrivée de physiciens en biologie, le modèle de l'ADN a pu ensuite se développer et les gènes, issus des théories de l'hérédité du XIXe siècle et de la 
génétique du début du $\mathrm{xx}^{\mathrm{e}}$ siècle, ont pu trouver leurs places sur la chaîne hélicoïdale des nucléotides. En cette toute fin du $x^{\mathrm{e}}$ siècle, le concept de gène, toujours très utilisé et très utile, ne semble pourtant pas s'être précisé. Au contraire, dès les années soixante, on assiste à une déconstruction du concept de gène par les biologistes moléculaires, les généticiens des populations continuant jusqu'à aujourd'hui d'utiliser une conception du gène antérieure à l'avènement de la biologie moléculaire. Ce concept, relativement nouveau dans l'histoire, est né dans un contexte précis, celui du début $\mathrm{du} \mathrm{xx}^{\mathrm{e}}$ siècle. Il exprime généralement l'idée d'un lieu spécifique de l'hérédité présent dans l'organisme, tel un déterminant matériel qui assure la transmission des caractères. Il est ainsi caractéristique de l'approche réductionniste des phénomènes vitaux défendue par certains biologistes de la seconde moitié du $\mathrm{XIX}^{\mathrm{e}}$ siècle. Il a été établi non seulement pour expliquer la transmission des caractères mais aussi le processus de l'évolution (modification et apparition des espèces, rôle des circonstances extérieures, etc.) et le développement embryonnaire particulièrement étudiés alors. Il a répondu également aux demandes adressées aux scientifiques de rationalisation des processus de sélection venant des améliorateurs d'espèces végétales et animales utiles. La recherche de modèles particulaires pour expliquer l'hérédité et les phénomènes connexes, expression de la volonté de rupture avec le vitalisme - rupture qui se développe à partir de la seconde moitié du XIX ${ }^{\mathrm{e}}$ siècle - aboutit à la mise en place de la notion de gène puis à la constitution de la biologie moléculaire. Elle a ainsi induit le succès de l'approche réductionniste et la primauté du choix du niveau macromoléculaire (entre organisme et atome) en biologie au $\mathrm{xx}^{\mathrm{e}}$ siècle $^{20}$. Le gène, la génétique, la biologie moléculaire et ses modèles ont une forte historicité et sont d'abord l'expression d'une époque. Le gène n'a pas été donné à l'homme, c'est l'homme qui l'a créé. Pourtant, la littérature, notamment le roman de science-fiction, présente le gène comme un être précis bien réel, implicitement présent avant sa "découverte" et définitivement existant, partie de la "nature". L'histoire du concept de gène, de sa représentation et de son utilisation, est exemplaire de ce fait de l'histoire des sciences. On est surpris que la circulation du sang n'ait été admise qu'au $x_{\text {VII }}{ }^{\mathrm{e}}$ siècle et qu'Harvey ait été présenté

20. Voir notamment Michel Morange, La part des gènes, Paris, Odile Jacob, 1998; Gilles Denis, "Gène", dans Dominique Lecourt, Dictionnaire d'bistoire et philosophie des sciences, Paris, Presses universitaires de France, 1999. 
comme un "génie" pour l'avoir fait. Cependant, Harvey ${ }^{21}$ n'était préoccupé initialement que d'attester que les aristotéliciens avaient raison contre les galiénistes en montrant que du sang se trouve nécessairement à la fois dans les artères et dans les veines et non pas uniquement dans ces dernières. Il ne s'interrogeait pas sur l'idée de circulation. Continuité et discontinuité sont en histoire étroitement imbriquées. Toutefois, lorsque l'on étudie en détail un objet de science d'une autre époque, même peu éloignée de la nôtre (théorie enzymatique de la vie par exemple), on se sent dépaysé et c'est la discontinuité qui semble l'emporter. Le dépaysement se réduit lorsque l'on se familiarise avec cette époque comme lorsque l'on finit par s'accoutumer à un pays qui nous est étranger. Une fiction, qui se déroule dans un futur éloigné ou un autre monde et qui s'appuie sur la génétique ou la biologie moléculaire contemporaine, restera finalement dans l'histoire de la littérature comme l'expression de l'époque de son auteur, ce qui est somme toute cohérent mais porteur d'une vision "réaliste", "universalisante", "ampliative" de la science. D'une manière plus générale, la littérature diffuse dans le grand public les concepts de la science, aidant celle-ci à imposer sa "nature" et sa "méthode" en les faisant "connaittre". En restant lucide sur notre capacité à nous défaire de nous-même, on peut cependant tenter de proposer d'autres sciences pour raconter notre futur ou même d'autres manières d'appréhender (expliquer-vivre avec) le monde (naturecosmos). On peut aussi s'amuser à figurer le futur en s'appuyant sur des théories passées, par exemple la médecine galiéniste et la physique aristotélicienne, ou sur des modèles créés dans d'autres civilisations (chinoise, indienne, pré-colombienne, etc.). Certains auteurs de science-fiction le font mais très généralement de manière très partielle, la science contemporaine restant la référence principale.

\section{Le roman policier}

Un autre discours littéraire est parfois étudié pour éclairer les rapports entre littérature et science. Il s'agit du roman policier dont la trame est l'intrigue, l'élaboration d'hypothèses, leur mise à l'épreuve, le rassemblement de faits, leur articulation logique. Il

21. Voir notamment Jerome J. Bylebyl, "Nutrition, Quantification and Circulation ", Bulletin d'histoire médicale, n ${ }^{\circ}$ 51, 1977, p. 369-385. 
semble suivre ainsi la méthode scientifique inductive, avec ses aspects aléatoires mais néanmoins sa démarche rigoureuse, planifiée, ses hypothèses testées par l'expérience et à l'épreuve des faits, telle que le grand public habituellement se l'imagine. Ce dernier et certains auteurs, qui analysent les romans policiers dans leur rapport avec la science, confondent ainsi fréquemment, lorsqu'ils parlent de "démarche scientifique", la marche chronologique, historique de la "science en action" et la marche reconstruite de la "science achevée". La première, alogique, aboutit à la création (d'objets, de concepts, d'explications, de modèles, de théories, etc.) et in finæ à l'article et à l'explication scientifique. Elle inclut par exemple les méandres de la pensée, la formation du savant et les diverses influences qu'il a reçues, les pressions idéologiques, sociales et administratives, les moyens matériels et financiers, les écrits au jour le jour jusqu'aux différents brouillons du texte à publier. L'écriture de ce dernier participe à "l'achèvement créatif". Le nouveau concept, la nouvelle explication émerge lentement à différents moments de ce processus intégrant le travail d'écriture du texte (ou des textes) à publier ${ }^{22}$. Holmes, par exemple, a observé l'évolution de la pensée de Lavoisier dans ses carnets de laboratoire, ses nombreux brouillons aboutissant à son texte sur la respiration des animaux lu à l'Académie royale des sciences de Paris en $1776^{23}$ et édité en 1780 dans les Mémoires de celle-ci ${ }^{24}$. Holmes y voit la "théorie de la respiration" émerger, se construire, se modifier, se préciser et s'éloigner. La seconde marche est une mise en forme logique, et se trouve présentée comme une méthode dans le texte (ou les textes) et l'explication scientifique achevés (hypothèse, matériel et méthode, résultats, conclusion, etc.) mais n'existe pas en acte ${ }^{25}$. La démarche scientifique décrite dans

22. Voir Frederic L. Holmes, Claude Bernard and Animal Chemistry. The Emergence of a Scientist, Cambridge, Harvard University Press, 1974; Frederic L. Holmes, Lavoisier and the Chemistry of Life, Madison, Wisconsin Press, 1985; Frederic L. Holmes, "Scientific Writing and Scientific Discovery", ISIS, $\mathrm{n}^{\circ} 78,1987$, p. 220-235.

23. Antoine Laurent Lavoisier, Expériences sur la décomposition de l'air dans le poumon des animaux, novembre 1776, Manuscrit, Papiers Lavoisier, Fiche 1349, Archives de l'Académie des sciences.

24. Antoine Laurent Lavoisier, "Expériences sur la respiration des animaux et sur les changements qui arrivent à l'air en passant par leur poumon ", Mémoires de l'Académie royale des sciences de Paris, Paris, Imprimerie royale, 1777 [1780], p. 185-194.

25. Paul Braffort, op. cit., p. 275-276. 
un texte scientifique publié exposant une "découverte" diffère de la démarche scientifique telle qu'elle a eu lieu pour l'obtenir ${ }^{26}$. "[Les articles scientifiques] fournissent une image de l'activité scientifique qui s'ajuste à une idéologie plutôt qu'à une pratique réelle." 27

Ce n'est pas seulement le cas des articles scientifiques. La pratique de la science est souvent assimilée dans la littérature au récit du texte scientifique. Pour tenter peut-être une pacification, certains préfèrent séparer comme deux activités différentes (mais non inconciliables) la "science en action" de la "science achevée" en disant que l'une participe du contexte de "découverte" et l'autre de celui de "justification" ou de "discours" 28 . Lorsque l'on assimile le développement de l'intrigue "à rebrousse-temps " et la participation du lecteur à la résolution de l'énigme, caractéristiques du roman policier traditionnel, à la "démarche scientifique traditionnelle", parle-t-on de l'ensemble du processus aboutissant à la rédaction de l'article scientifique ou simplement de la présentation reconstruite qui en est faite dans celui-ci? Suit-on les dires des savants sur ce qu'ils font dans leurs écrits scientifiques, reprend-on les définitions établies par certains philosophes de la méthode scientifique (Popper, etc.), s'appuie-t-on sur les analyses des historiens ou des sociologues des sciences (Latour, etc.) ou propose-t-on une autre représentation de la science? Ceux qui, philosophes, sociologues, historiens, débattent sur la nature de la science et de l'activité scientifique, n'arrivent pas à une conclusion commune, à une représentation unanime. Or la littérature, écho du monde, semble avoir une représentation somme toute banale. Le déroulement de l'intrigue du roman policier diffère essentiellement de la démarche scientifique par le fait que généralement les pièces à conviction et le meurtrier à découvrir existent, tandis que les "objets" (concepts, modèles, théories, etc.) "découverts" par le savant sont une proposition imaginée par lui pour "représenter-expliquer" un ensemble de phénomènes. Dans

26. Peter B. Medawar, The Art of the Soluble, Londres, Methuen \& co LTD, 1968, p.151; Frederic L. Holmes, op. cit.; Frederic L. Holmes, "Argument and Narrative in Scientific Writing", dans Peter Dear, op. cit., p. 164-181.

27. Medawar, cité par Holmes, op. cit., p. 221.

28. Voir notamment les textes cités de Holmes; Gerald Holton, L'imagination scientifique, Paris, Gallimard, 1981; Jaakko Hintikka et Fernand Vandamme, Logic of Discovery and Logic of Discourse, New York, Londres, Ghent, Plenum Press, 1985. 
ce sens, le roman policier est plus "réel" que la création scientifique.

\section{Histoire des sciences et littérature}

L'histoire des sciences cherche à analyser en détail comment se construit la science, ce qui la nourrit et ce qui produit cette dernière. Ses résultats lui servent dans les débats sur la nature de celle-ci. Elle s'intéresse notamment au processus qui aboutit à l'édification du texte édité, particulièrement lorsqu'il est porteur de nouvelles explications théoriques et d'une nouvelle représentation (réarrangement) de la nature.

\section{La spécificité du texte savant contemporain}

Le texte savant contemporain qui réinvente le processus créatif sur une base inductive s'est mis en place peu à peu depuis le $\mathrm{XvI}^{\mathrm{e}}$ siècle. On voit s'établir des normes qui se généralisent graduellement ${ }^{29}$. Prenons un exemple au milieu du xvIII ${ }^{\mathrm{e}}$ siècle, époque où la forme du texte scientifique est encore très variée, touchant à l'émergence des sciences de l'agriculture, domaine qui pourrait sembler éloigné de l'évolution perceptible dans les sciences physiques. Dans les tomes II et IV de son Traité de la culture des terres, Duhamel du Monceau présente d'une manière relativement différente les mêmes expériences sur cette maladie qui transforme la farine des grains de blé en une poussière noire. Dans le premier tome, il écrit en 1753:

Madame de Rouville, qui a ses terres dans notre voisinage, avait beaucoup recueilli de noir en 1750 dans les blés de la ferme de la Roche; elle fit ses semailles pour 1751, partie avec les blés de la récolte, qui, comme nous venons de le dire, étaient mêlés

29. Claire Salomon-Bayet, L'institution de la science et l'expérience du vivant. Méthode et expérience à l'Académie royale des sciences, 1666-1793, Paris, Flammarion, 1978; Andrew E. Benjamin, Geoffrey N. Cantor et John R. R. Christie, The Figural and the Literal. Problems of Language in the History of Science and Philosophy (1630-1800), Manchester, Manchester University Press, 1987; Laurence J. Prelli, A Rhetoric of Science. Inventing Scientific Discourse, Columbia, S. C., University of South Carolina Press, 1989; Christian Licoppe, La formation de la pratique scientifique. Le discours de l'expérience en France et en Angleterre (1630-1820), Paris, La Découverte, 1996. 
de noir, et partie des blés qui n'étaient point noirs, qu'elle avait tiré de Digny, qui est éloigné d'environ deux lieues de la Roche: l'un et l'autre blé avaient été passés à la chaux. En 1751, il s'est trouvé beaucoup de noir dans les terres qui avaient été ensemencées avec les grains recueillis sur ce lieu: mais il y en avait fort peu dans les terres ensemencées avec les blés de Digny. Cette expérience est bien propre à faire croire que le noir est une maladie héréditaire. ${ }^{30}$

Nous sommes ici près d'un récit champêtre. Dans le quatrième tome, le récit se fait plus concis, plus formel. Il est annoncé par un sous-titre évocateur:

Première expérience.

Un de nos voisins avait tous les froments de sa récolte fort infectés de noir; il n'ensemença qu'une partie de ses terres avec ce grain exempt de noir, qu'il tira de deux lieux de chez lui. Il ne s'est trouvé que dans les terres qu'il avait ensemencées avec son propre grain. Cette expérience prouve donc que les maladies dont il s'agit sont héréditaires. ${ }^{31}$

Le texte est déjà une reconstruction, une mise en forme, sur une base inductive, du processus aboutissant à la "découverte". Il se rapproche de nos écrits scientifiques mais s'éloigne du récit décrivant l'émergence de l'explication de "maladie héréditaire". Le premier texte, bien que davantage exhaustif, ne représente guère non plus ce récit que Duhamel n'a sans doute pas les moyens d'établir. Il devrait pour cela notamment montrer comment et pourquoi à ce moment-là les "savoirs" et "pratiques" paysans sont l'objet d'études de la part des auteurs ruraux, notables de campagne, et des physiciens-agriculteurs, savants se mettant au service de "l'art" agricole, d'où la reconnaissance du rôle des semences bien connu par les cultivateurs. Il devrait aussi étudier les transferts de modèles issus de la médecine, expliquer pourquoi les physiciens-agriculteurs sont essentiellement des chimistes et ainsi ces modèles essentiellement chimistes, d'où la notion, pour le XVIII ${ }^{\mathrm{e}}$ siècle, de "maladies héréditaires" 32 . Le mo-

30. Henri Louis Duhamel du Monceau, Traité de la culture des terres suivant les principes de M. Tull, anglois, tome second, Paris, H. L. Guérin et L. F. Delatour, 1753, p. 170.

31. Henri Louis Duhamel du Monceau, Traité de la culture des terres, tome quatrième, Paris, H. L. Guérin et L. F. Delatour, 1755, p. 214.

32. Voir Gilles Denis, "Agronomie, chimie et botanique (1755-1805) en France/ L'exemple des maladies des plantes: principe chimique ou plante 
dèle finalement retenu a pour origine les "savoirs" et les "pratiques" paysans, les modèles de la maladie issus de la médecine et de la chimie, l'idéologie "utilitariste" et son désir de lier "expérience" des ouvriers et celle des savants, l'engouement pour la science de type newtonnienne, l'approche physiocratique (trois mouvements en œuvre à ce moment-là dans la "république des lettres"), l'acculturation et l'alphabétisation des campagnes. Il pourrait être rétorqué que ce qui compte in fince c'est la "découverte" de l'explication de la maladie. Le problème est qu'elle ne tiendra, bien que largement utilisée dans les Instructions royales, qu'une cinquantaine d'années lorsque les naturalistes répondront à la question selon leurs méthodes et leur tradition. Cette explication de la maladie ne semble donc exister que dans le cadre d'un contexte précis.

\section{Les frontières entre les disciplines}

L'histoire des sciences cherche à préciser l'évolution de la notion de science, la façon de se représenter l'activité scientifique, la hiérarchie et les frontières des disciplines savantes, entre elles et les autres activités et réalisations humaines. Si au Moyen Âge, arabe puis latin, la musique et les mathématiques sont associées dans l'enseignement, l'histoire des sciences montre que ce n'est pas parce qu'alors régnait une vision plus œcuménique que la nôtre des rapports entre arts et sciences. Elle constate que les objets étudiés par les différentes disciplines le sont simplement sur d'autres bases, qu'ils sont ainsi d'une autre nature et que le découpage des disciplines obéit ainsi à une autre logique. La musique appartient alors aux mathématiques. Selon Ibn Sina (Avicenne) par exemple, d'inspiration aristotélicienne, la philosophie se divise en une partie théorique (dont la fin est le vrai) et une partie pratique (dont la fin est le bien). Les parties de la philosophie théorique sont au nombre de trois: la science inférieure

microscopique parasite", dans Biology Integrating Scientific Fundamentals, Munich, Institut für Geschichte der Naturwissenschaften, 1997; Gilles Denis, "Transmission des idées savantes sur les maladies des plantes à travers les textes grecs, latins, arabes puis modernes", dans Méditerranée, monde rural et histoire des sciences, Perpignan, Presses de l'Université de Perpignan, 1998; Gilles Denis, "Les pratiques paysannes dans l'émergence de l'agronomie au $\mathrm{XVIII}^{\mathrm{e}}$ siècle. Le cas des débats sur la transmission des maladies des blés", Revue d'histoire des sciences, édition en cours. 
(la physique), la science intermédiaire (la science mathématique), la science supérieure (la métaphysique). Dans la seconde, la science mathématique, on trouve quatre parties: la science des nombres, la géométrie, l'astronomie et la musique. On observe des classifications similaires chez de nombreux auteurs et dans l'enseignement en Europe, notamment à Paris, au milieu du $\mathrm{XIII}^{\mathrm{e}}$ siècle. Ainsi, mathématique et musique se trouvent liées dans l'enseignement parce que la seconde est une partie de la première ${ }^{33}$. Ce n'est pas la preuve que science et non-science sont liées, sont proches ou se nourrissent l'une l'autre (ce n'est pas non plus la preuve du contraire). Nous sommes simplement en présence d'une classification des sciences (de la connaissance) qui n'est plus la nôtre, dans laquelle les notes de musique sont beaucoup plus proches des nombres que ne le sont, par exemple les "mouvements" des corps (notamment célestes). Ce qui est l'inverse dans notre classification depuis que la physique n'est plus qualitative mais mathématisée et la musique émancipée.

Il est d'usage de parler, pour les auteurs du xvIII ${ }^{\mathrm{e}}$ siècle, de leur esprit (approche) "encyclopédique", non pas dans le sens inspiré par le mouvement né de l'Encyclopédie de Diderot et d'Alembert, mais dans le sens "qui embrasse l'ensemble des connaissances". Parallèlement, on présente parfois l'évolution ultérieure comme celle de la spécialisation et du renfermement disciplinaire même si on décrit parfois aussi la seconde moitié du $\mathrm{xx}^{\mathrm{e}}$ siècle comme "une époque où nombreux sont les professionnels de l'interdisciplinarité " 34 . Une partie de cette reconstruction historique s'appuie selon nous sur une vision anachronique du découpage des savoirs et des centres d'intérêt qui leur sont attachés. Au premier abord, les savants du xvIII ${ }^{\mathrm{e}}$ siècle peuvent nous sembler se consacrer à tout. Par exemple Mathieu Tillet, métallurgiste, directeur de la monnaie à Troyes, directeur puis trésorier de l'Académie royale des sciences de Paris, précepteur pour les questions d'agriculture du Dauphin sous Louis XVI, étudie la ductilité des métaux, la nature des terres et la maladies

33. Voir notamment Rabia Mimoune, "Épitre sur les parties des Sciences intellectuelles d'Abu Ali al-Husayn Ibn Sina" et Édouard Wéber, "La classification des sciences selon Avicenne à Paris vers 1250 ", dans Jean Jolivet et Roshdi Rashed, Études sur Avicenne, Paris, Les belles lettres, 1984, respectivemment p. 143-151 et 77-101.

34. René Thom, "La modélisation des processus mentaux", dans Fonctions de l'esprit. Treize savants redécouvrent Valéry, Paris, Hermann, 1983, p. 193. 
du "noir" des blés (carie). Nous pourrions dire qu'il est, selon nos critères, ingénieur des mines, chimiste, agronome, naturaliste, physiologiste, administrateur, professeur et chercheur. En fait, lorsque l'on étudie en détail ses textes, on se rend compte qu'il appartient à une communauté de chimistes partisans de "l'utilité des sciences" qui analysent de nombreux phénomènes de la nature de la même manière. Les différents métaux se forment dans la terre par fermentation. Il en est de même des différentes terres dont la culture permet plus ou moins une fermentation à l'origine de leur fertilité. Le "noir" est dû à une fermentation anormale sous l'action d'un "virus" (poison) agissant comme "ferment" (ou "levain") et en modifiant la sève qui se déverse dans le grain en une poussière noire puante. Le ferment normal habituel qui modifie cette sève en farine blanche est alors empêché d'agir. Tillet suit ici l'explication de la syphilis donnée par Jean Astruc qui fait aussi appel aux notions de ferment et de fermentation. Nous pourrions citer également l'apothicaire Parmentier, le médecin iatrochimiste Fourcroy ou Chaptal. Tous tentent d'utiliser la chimie pour expliquer un grand nombre de phénomènes qui leur apparaissent de même nature malgré les différents lieux où ils s'observent (mines, ateliers, champs, laiteries, reins et foies, etc.); phénomènes que nous pourrions regrouper sous le terme aristotélicien de "sublunaires", à savoir les phénomènes soumis à la génération et à la corruption (par opposition au monde parfait, au mouvement circulaire et sans corruption des astres). Ces auteurs n'ont pas le sentiment de travailler sur des objets de provenances disciplinaires différentes. Ils constituent en quelque sorte une communauté et une discipline. Un autre exemple: Duhamel du Monceau s'intéresse à la construction navale, à la physiologie végétale et à la culture des terres. Il s'inscrit d'une manière extrême dans le mouvement utilitariste (Condorcet le désignera comme "l'apôtre de l'utilité des sciences") et combat explicitement la spéculation scientifique. Il écrit en faveur des thèses physiocratiques. Il semble, pour un observateur du $\mathrm{xx}^{\mathrm{e}}$ siècle, s'intéresser lui aussi un peu à tout. Mais en le connaissant mieux, on peut l'imaginer nous dire qu'il se limite à aborder les questions pratiques qui posent problème à l'enrichissement du royaume et de ses habitants. De la même manière, par exemple, un percepteur d'aujourd'hui récolte les participations aux recettes de l'État aussi bien d'un ménage de boulanger que d'une grande entreprise de l'automobile sans pour autant être taxé d'esprit encyclopédiste même si de plus il donne 
des cours et écrit un livre pour formuler son point de vue sur son travail et sa place dans l'économie. Il existe bien entendu de nombreux auteurs, notamment savants, du xviII ${ }^{\mathrm{e}}$ siècle qui s'intéressent à des thèmes éloignés d'un point de vue disciplinaire. Il existe aussi des auteurs, tel Humboldt au tournant du siècle, qui sont clairement en quête d'une unité des savoirs. Cette diversité d'intérêts doit être néanmoins distinguée d'un regard anachronique sur le découpage des savoirs et des engagements professionnels. N'oublions pas que la plupart des scientifiques des deux siècles suivants, ceux qui entre autres ont laissé leurs marques au $\mathrm{xx}^{\mathrm{e}}$ siècle (Ernst Mach, Henri Poincaré, Albert Einstein, Niels Bohr, Louis de Broglie, Jacques Monod, etc.), ont montré des préoccupations littéraires, philosophiques ou/et politiques qui se sont exprimées au sein de leurs écrits ${ }^{35}$. Il faut aussi ajouter que l'historien des sciences apprend à se familiariser avec l'hétérogénéité des époques du passé, à décrire la diversité de l'activité scientifique derrière les grands noms retenus par l'histoire. La science ne se fait pas dans tous les lieux de la même manière. La séparation entre les disciplines est beaucoup plus marquée dans les Mémoires de l'Académie royale des sciences de Paris que dans le Journal historique sur les matières du temps où s'expriment dans de petites dissertations des lettrés de toute la France sur diverses questions scientifiques, littéraires ou autres. Les rapports entre les différentes sciences, entre celles-ci et les "arts" (au sens du XvIII ${ }^{\mathrm{e}}$ siècle, nous dirions aujourd'hui "technologies"), entre celles-ci et les autres domaines (littérature, poésie, etc.) ne sont pas perçus de la même manière par tous les auteurs, par toutes les institutions. Pour certains tenants de l'utilité de la seconde moitié du XvIII ${ }^{\mathrm{e}}$ siècle, tels Francis Home, Fourcroy ou

35. Ernst Mach, La connaissance et l'erreur, traduction abrégée de l'allemand par Marcel Dufour, Paris, Flammarion, 1908. Henri Poincaré, La science et l'bypothèse, Paris, Flammarion, 1968. Albert Einstein et Sigmund Freud, Pourquoi la guerre? Why war?, Paris, Institut international de Coopération intellectuelle, 1933. Albert Einstein, Comment je vois le monde, Paris Flammarion, 1934. Niels Bohr, "Discussion with Einstein on epistemological problems in atomic physics", dans Paul Arthur Schilpp, Albert Einstein. Philosopher-Scientist, Lassalle, Illinois, Open Court, 1949. Louis de Broglie, Certitudes et incertitudes de la science, Paris, Albin-Michel, 1966. Louis de Broglie, "Déduction et induction dans la recherche scientifique", "La culture scientifique suffit-elle pour faire un homme?", dans Louis de Broglie, Un itinéraire scientifique, textes réunis et présentés par Georges Lochak, Paris, La Découverte, 1987, respectivement p. 197-200 et p. 201-205. Jacques Monod, Le hasard et la nécessité, Paris, Éditions du Seuil, 1971. 
Reynier, la chimie est une science modèle car elle est proche des "arts" (technologie), tandis que la botanique est à reléguer comme activité "oiseuse" au rang de la poésie. À la fin du siècle, la botanique apparaît au contraire, notamment sous l'influence de Rousseau, du côté du savoir populaire contre la spéculation mathématique des institutions académiques ${ }^{36}$.

\section{Le sens des mots et l'évolution des concepts savants}

D'une façon générale, la signification des termes scientifiques évolue relativement vite. La notion de virus, par exemple, de l'an 2000 diffère, après plusieurs années de recherche sur le SIDA, de celle des années quatre-vingt. Nous avons vu qu'au XvIII siècle, ce terme est synonyme de celui de "poison". La syphilis se maintient en Europe, selon Astruc, par un "levain étranger" qui se "renouvelle toujours". Le "levain, venin, virus vérolique" se multiplie par degrés dans le corps en transformant les plus petites particules du "fluide" (sang et autres humeurs) sur lequel il agit en particules semblables aux siennes, de la même manière "qu'un levain ordinaire fait lever plusieurs livres de pâte; et que chaque livre de cette pâte en fait lever plusieurs autres." Au début du $\mathrm{xx}^{\mathrm{e}}$ siècle, le "virus" désigne la substance restant contagieuse malgré le passage de l'isolat la contenant à travers un filtre chargé de retenir les bactéries. Dans les années trente, après plusieurs travaux cherchant à isoler la substance contagieuse de la mosaïque du tabac, supposée être une protéine, un "virus" donc, celui-ci est identifié comme une nucléoprotéine par Bawden et Pirie. Les recherches sur les maladies virales à partir de ces années ont contribué à la construction de la biologie moléculaire. Aujourd'hui, à la suite de ces travaux, le "virus" est assimilé à un morceau de chromosome et à un organisme vivant ayant besoin d'une cellule hôte pour se multiplier et survivre. Nous pourrions étudier, avec les mêmes constats, de nombreux termes savants tels que ceux de gène, microbe, parasite, vie, électron, force, énergie, éther, sel, âme, etc. ${ }^{37}$. La charge poétique de certains de ces mots (âme, éther, etc.) peut s'en trouver modifiée dans les

36. Voir Gilles Denis, les différents textes cités.

37. Voir Scott Walter, "Éther", Gilles Denis, "Gène", Federic L. Holmes, "Sel", dans Dominique Lecourt, Dictionnaire d'histoire et de philosophie des sciences, op. cit. 
30

textes littéraires lorsque l'on identifie la part du regard anachronique. L'histoire des sciences doit servir ainsi à l'étymologie et à l'histoire des mots. Nos travaux sur l'histoire des représentations savantes des maladies des plantes cultivées nous ont permis de mettre à jour tout un vocabulaire disparu et de proposer des hypothèses sur l'origine de certains termes restée en partie inconnue dans les recherches étymologiques. Parmi de nombreux exemples, citons les "rosées mielleuses", les "rosées farineuses", les "miellats", les "emmielures" qui sont des sortes de rosées non aqueuses retombées sur les plantes (selon la météorologie des $\mathrm{XVII}^{\mathrm{e}}$ et $\mathrm{XVIII}^{\mathrm{e}}$ siècles) qui provoquent des fermentations sur elles. À la fin du XIX ${ }^{\mathrm{e}}$ siècle, ces termes seront oubliés. Lorsqu'il s'agira pour les scientifiques français de désigner certaines de ces maladies qu'ils étudient et pour lesquelles on accuse alors un cryptogame microscopique, ils utiliseront un terme anglais équivalent, "mildew" (rosée de miel) qui devient "mildiou". Au sujet de l'ergot, maladie du seigle (grains noirs à l'extérieur et allongé en une forme de corne), les dictionnaires étymologiques soit reconnaissent que l'origine est inconnue, soit prétendent que "ergot" viendrait d'un radical préroman qui désigne une "chose pointue". Le Robert écrit que la première apparition écrite date de 1440 sous la forme "ergos" et de 1175 sous la forme "argoz" 38 . L'étude des termes utilisés pour désigner les maladies des plantes depuis l'Antiquité nous a permis d'identifier deux termes en latin: "rubigo" et "cerugo". Le premier désigne au sens propre la rouille du fer, le second la rouille d'airain, rouilles des cuivres ("aeris", cuivre et bronze). Ces deux termes servent aussi à désigner des maladies des grains, le premier étant beaucoup plus largement utilisé. Dans le texte latin de la Vulgate on trouve ces deux termes, selon les versets, pour désigner une des calamités tombant sur la terre. Ils sont traduits dans les bibles de langue française, suivant les époques, notamment par "mielach", "rouillure", "nielle", "rouille". Le terme "nielle" est en effet un des termes les plus utilisés en français jusqu'au XviII ${ }^{\mathrm{e}}$ siècle pour désigner les maladies des grains de céréales. Toutes les rouilles des métaux et des plantes sont perçues par les savants, de l'Antiquité jusqu'au $\mathrm{XVIII}^{\mathrm{e}}$ siècle, comme le dépôt ou l'action corrosive d'une substance présente dans l'air. Jean Ruel utilise le

38. Paul Robert, Dictionnaire alphabétique et analogique de la langue française, Paris, le Robert, 1987. 
terme "aerugo" pour la "nielle" de l'orge. Dans son De Stirpibus Epitome, il donne une liste en latin, avec traduction française des simples. On y trouve donc différentes plantes, mais aussi deux "aerugo", celui des cuivres, et celui de l'orge ${ }^{39}$ :

Aerugo, uiride aeris medicis, vertd grys

et

Aerugo in hordeo, bledz neislez. ${ }^{40}$

Je pose l'hypothèse que le terme "ergot", désignant le "grain cornu" (le seigle, parfois le blé, ergoté), ait pour origine "aerugo", de la même manière que la "rouille" des blés a pour origine "rubigo". Il faut noter la couleur brunâtre, noirâtre, relativement proche de celle de la "rouille d'airain" relativement à celle roux-orangé de la rouille des feuilles des céréales et du fer. On utilise, en français, à l'époque moderne, le mot "aerugineux", "airugineux", "erugineux", dans les textes savants afin de désigner la couleur de "rouillure d'airain". Ainsi Ambroise Paré écrit ${ }^{41}$ :

"La cholere airugineuse ayant couleur de vert-de-gris"

"Un virus écumeux, fétide, virulent et erugineux, c'est-à-dire de couleur de rouilleure d'airain. "

Selon La Quintinye, jardinier de la fin du xvir ${ }^{\mathrm{e}}$ siècle, on appelle "argot" l'extrémité d'une branche qui est morte, et, que lorsqu'on coupe cette extrémité "jusque sur le vif ", d'après lui, on dit "Ôter l'argot"; extrémités des branches ${ }^{42}$. On peut penser à l'aspect pointu d'une extrémité d'une branche qui aurait perdu ses feuilles ou à l'aspect brunâtre verdâtre, bronze, plomb de certains symptômes. Remarquons que l'on trouve parfois au XVIII ${ }^{\mathrm{e}}$ siècle, à la place de "nielle" et "niellé", pour désigner une maladie des grains, le terme "bronze" et "bronzé". Ajoutons que

39. Jean Ruel, In Ruellium de Stirpibus Epitome, Parisiis, Apud Ioannem Ludoicum Tiletanum, 1544, (première édition: 1539).

40. Termes latins ou de vieux français que nous pourrions traduire par:

Rouille d'airain, vert de l'airain pour les médecins, vert-de-gris

et

Rouille d'airain dans l'orge, grains niellés (notre traduction).

41. Cité par Huguet, Dictionnaire de la langue française du XvI ${ }^{e}$ siècle, Paris, Honoré, 1928.

42. Jean de La Quintinye, "Explication des termes du jardinage", dans Instruction pour les jardins fruitiers et potagers, Paris, La compagnie des libraires, 1700 ; cf. Tome I, p. 67-134. 
32

certaines maladies des arbres sont désignées aujourd'hui par le mot "plomb".

\section{Conclusion}

L'histoire des sciences s'intéresse à la science au jour le jour; toute la science, depuis la formation des savants jusqu'aux textes diffusés, des influences idéologiques jusqu'aux locaux des laboratoires. L'histoire des sciences, d'une certaine manière, déloge la science de son piédestal pour la ramener aux niveaux des autres activités humaines. La "découverte", la "nouveauté" n'apparaît plus comme un don reçu par le savant qui suit la bonne méthode (expérimentale et/ou intellectuelle) mais comme une construction lente, tortueuse et laborieuse de la coalition d'un contexte et d'une tradition intrinsèque (intellectuelle et/ou institutionnelle) au niveau du savant ou d'une équipe. La représentation de l'activité scientifique (ses nourritures et ses produits) qui en découle diffère ainsi de celle apparaissant dans les textes de la science achevée. La littérature et l'analyse littéraire doivent tenir compte de cette dichotomie.

Par quelques exemples, nous avons cherché à montrer l'intérêt pour l'analyse littéraire de s'appuyer notamment sur les acquis de l'histoire des sciences: histoire et étymologie des mots, concepts et termes remis dans leur contexte, évolution du formalisme des textes savants, évolution des frontières disciplinaires et des représentations que les auteurs savants en ont, hétérogénéité de la science et diversité des opinions à ce sujet à chaque époque, etc.

Il ne faudrait pas pour autant dissimuler le fait que l'histoire des sciences est en quelque sorte une reconstruction logique, une narration compréhensible, à partir d'un choix de sources et d'événements, de remplissage pour les lier ensemble, d'apriori et d'une multitude d'autres éléments plus ou moins exprimables. L'historien propose un récit qui "explique", c'est-à-dire simplement qui "[montre] le déroulement de l'intrigue, le fait comprendre", comme le fait un roman. L'explication n'est pas à prendre, si l'on suit Paul Veyne, dans un sens fort où "expliquer" veut dire "assigner un fait à son principe ou une théorie à une théorie plus générale":

[L'histoire] demeure fondamentalement un récit et ce qu'on nomme explication n'est guère que la manière qu'a le récit de 
s'organiser en une intrigue compréhensible [...]. Puisque telle est la quintessence de l'explication historique, il faut convenir qu'elle ne mérite pas tant d'éloges et qu'elle ne se distingue guère du genre d'explication qu'on pratique dans la vie de tous les jours ou dans n'importe quel roman où l'on raconte cette vie; elle n'est que la clarté qui émane d'un récit suffisamment documenté; elle s'offre d'elle-même à l'historien dans la narration et n'est pas une opération distincte de celle-ci, pas plus qu'elle ne l'est pour un romancier ${ }^{43}$.

Le récit de l'histoire des sciences, le texte scientifique pour diffusion de la science achevée, constituent deux formes d'écriture parmi d'autres et appartiennent de ce fait à la littérature comme le roman policier ou la poésie. Ils obéissent tous les deux à des normes précises. Il y a une mise en sens historique, scientifique, comme il y a une mise en sens poétique, romantique ou érotique. Le récit d'histoire des sciences relate le vécu, qui s'appuie sur ce qui a "réellement eu lieu", l'histoire en action, mais en expliquant finalement peu. Le texte scientifique est une mise en forme logique. Il parle peu du vécu, "ce qui a réellement eu lieu ", mais cherche à expliquer fortement en proposant des modèles, en posant des principes, en offrant des théories.

43. Paul Veyne, Comment on écrit l'bistoire, Paris, Éditions du Seuil, 1979, p. 6771. 